\title{
P03-1-14 Poster session
}

\section{Blockade of GAPDH nuclear translocation in the hippocampus contributes to anti-depressant-like action in stressed mice via HPA axis}

\author{
Hidemitsu Nakajima', Sunao Nakamura ${ }^{1}$, Mitsuru Kuwamura², Yasu-Taka Azuma ${ }^{1}$, \\ Takatoshi Hikida $^{3}$, Tadayoshi Takeuchi ${ }^{1}$
}

${ }^{1}$ Laboratory of Veterinary Pharmacology, Graduate School of Life and Environmental Sciences, Osaka Prefecture University, Japan, ${ }^{2}$ Laboratory of Veterinary Pathology, Graduate School of Life and Environmental Sciences, Osaka Prefecture University, Japan, ${ }^{3}$ Laboratory for Advanced brain Functions, Institute for Protein Research, Osaka University, Japan

It has been recently reported that various biological stress has been associated with enhanced generation of reactive oxygen species (ROS) causing oxidative stress in the brain. Moreover, robust levels of oxidative stress in the brain has been implicated in stress-related neuropsychiatric disorders such as a major depression. But the underlying mechanisms are still unclear. Glyceraldehyde-3-phosphate dehydrogenase (GAPDH) is well known as a key enzyme of glycolysis, but several lines of evidence suggest that GAPDH is a multifunctional protein which has the strong linkage to oxidative stress. Here we aim to elucidate a role of GAPDH in stress-related neuropsychiatric disorders using restraint and water immersion (RWI) stress-models. In this model, mice showed the massive increase of ROS and nuclear translocation of GAPDH in hippocampal CA1 region and dentate gyrus. The knock-down of hippocampal GAPDH suppressed the RWI stress-induced changes, both reduction of body weight and increase in the weight of adrenal glands. Additionally, depression-like behavior assessed by tail-suspension test was significantly improved by the GAPDH knock-down. Further, we examined whether the treatment with CGP3466B, an inhibitor of GAPDH nuclear translocation, affects these depressive parameters, in comparison with clomipramine, a tricyclic antidepressant. The RWI stress-induced depressionlike behavior was ameliorated by these agents, while improvements of both body- and adrenal glands-weights were unique to treatment with CGP3466B. Moreover, an interaction of glucocorticoid receptor (GR) with GAPDH in the hippocampus was found and the RWI stress augmented its interaction, while treatment with CGP3466B significantly decreased the augmentation of GR-GAPDH interaction. These results suggest the significance of ROS-dependent GAPDH-nuclear translocation in the pathophysiology of stress-related neuropsychiatric disorders via HPA axis and could provide its novel therapeutic strategy. 